\title{
Stabilization in a Chemostat with Sampled and Delayed Measurements and Uncertain Growth Functions*
}

\author{
Frédéric Mazenc $^{\mathrm{a}} \quad$ Jérome Harmand $^{\mathrm{b}} \quad$ Michael Malisoff $^{\mathrm{c}}$ \\ ${ }^{a}$ EPI DISCO Inria-Saclay, Laboratoire des Signaux et Systèmes, CNRS, CentraleSupélec, Université Paris-Sud, \\ 3 rue Joliot Curie, 91192, Gif-sur-Yvette, France. \\ ${ }^{\mathrm{b}}$ LBE, INRA, 11100, Narbonne, France. \\ ${ }^{\mathrm{c}}$ Department of Mathematics, Louisiana State University, Baton Rouge, LA 70803-4918, USA.
}

\begin{abstract}
We provide a new control design for chemostats, under constant substrate input concentrations, using piecewise constant delayed measurements of the substrate concentration. Our growth functions can be uncertain and are not necessarily monotone. The dilution rate is the control. We use a new Lyapunov approach to derive conditions on the largest sampling interval and on the delay length to ensure asymptotic stabilization properties of a componentwise positive equilibrium point.
\end{abstract}

Key words: output feedback, stabilization, delay, sampling

\section{Introduction}

This work continues our search for controls that stabilize componentwise positive equilibria in chemostat models, under the incomplete state measurements and model uncertainties that usually occur in biotechnology laboratories, and so is strongly motivated by the ubiquity of the chemostat in a plethora of biological and engineering settings that are of compelling interdisciplinary interest, in which stabilization of componentwise positive equilibria is needed to ensure persistence of species. The chemostat is used for the continuous culture of microorganisms. It was first studied in Monod (1950) and Novick and Szilard (1950). It is regarded in biotechnology, ecology, and microbiology as an ideal way to represent cell or microorganism growth, wastewater treatment, or natural environ-

\footnotetext{
* Corresponding author: F. Mazenc. A preliminary version appeared in the proceedings of the 2016 American Control Conference; see the end of Section 1 for the differences between the conference version and this paper. Malisoff was supported by NSF-ECCS Grants 1102348 and 1408295 and Roy P. Daniels Professorship \#3 in the LSU College of Science. The first author acknowledges financial support from the MATH-AmSud project "Stability and Dichotomies in Differential and Delay Equations". Some of the third author's work was done while he visited the US National Science Foundation in Arlington, VA. He appreciated their hospitality during the period. The authors thank Paul Varnell for assisting with the simulations.

Email addresses:

frederic.mazenc@12s. centralesupelec.fr (Frédéric

Mazenc), Jerome.Harmand@supagro.inra.fr (Jérome

Harmand), malis off@lsu.edu (Michael Malisoff).
}

ments like lakes; see Beauthier et al. (2015); Bernard et al. (2001); Fritsch et al. (2015); Gouzé and Robledo (2005); Lemesle and Gouzé (2008), and Robledo et al. (2012). The variables are the microorganism and substrate concentrations, whose dynamics are based on mathematical models, e.g., mass-balance equations; see Mazenc et al. (2008) and Smith and Waltman (1995). Two challenges in designing controls for chemostats are their nonlinearity and their lack of online actuators and sensors; see Cougnon et al. (2011).

Moreover, when online devices are available to measure biomass and substrate concentrations, they usually only provide delayed discrete measurements. It is common to design controls using continuous time models, which are then discretized before being applied. However, to prove that continuous time controllers ensure that the desired stability objectives are met, one must show robustness with respect to discretization. Chemostats are also subjected to uncertainty in the growth functions, which should also be taken into account in the control design. To the best of our knowledge, no rigorous theoretical analysis in the literature has addressed the delay, robustness, and sampling problems that we consider here. The work Robledo (2009) assumes that the measurements are continuous.

The preceding remarks motivated Mazenc et al. (2013a) and this work, which solves a complementary problem to the ones in Mazenc et al. $(2013 a)$. Here we consider the classical chemostat model in Smith and Waltman (1995) that contains one substrate and one species, except here we also include delays, sampling, and uncertainties, which are three features that are not contained in the classical 
chemostat model. We assume that the input substrate concentration is constant, and that the growth rate is of Haldane type (which has a growth limitation for low substrate concentrations, and inhibition at high concentrations). The dilution rate is the control, and uses delayed and sampled observations. Controlling this system is difficult, for two reasons. First, works such as Mazenc et al. (2013b) that prove global asymptotic stability under delay and sampling use state feedbacks. Since our work has output feedbacks, it is outside the scope of Mazenc et al. (2013b).

Second, chemostats with non-monotonic growth rates generally have multiple equilibria, under constant dilution rates. One is unstable, while another is locally exponentially stable. The work Mazenc et al. (2013a) stabilized points of the second type, but here we stabilize points of the first type in cases where the growth rate is uncertain and not necessarily monotone. Our stabilizing controller only requires measurements of the substrate, which are piecewise constant and delayed. Under suitable bounds on the delay size and on the sampling interval, our control provides global asymptotic stability to a componentwise positive equilibrium when the growth function is known, and input-to-state stability (or ISS) (as defined in Khalil (2002)) with respect to uncertainties in the growth functions. This differs from Mazenc et al. (2013a), where no constraints on the delay and sampling intervals were used. We believe that these extra constraints are needed because under constant dilution rates, the equilibrium that we stabilize in this paper would have been unstable.

While reminiscent of Mazenc et al. (2013b), the barrier functions that we use here allow us to certify ISS, which was not considered in Mazenc et al. (2013b). The main result of Mazenc et al. (2013b) does not apply here, even in the special case where the growth functions are known. Our proof also differs from Mazenc and Malisoff (2010), which assumes that species measurements are available. When there are no perturbations, our results contrast with Gouzé and Robledo (2006) and other works that do not include delays or sampling or ISS. Our new work also improves on our conference version (i.e., Mazenc et al. (2016)), which did not allow uncertainties in the growth functions, because here, we prove ISS with respect to the uncertainties in the growth functions under arbitrarily large uncertainty bounds and positivity constraints on the states. See Section 3 below for our main result, Section 4 for its proof, and Section 5 for an illustration including simulations.

\section{Model and Notation}

Our basic chemostat model is

$$
\left\{\begin{array}{l}
\dot{s}(t)=D\left[s_{\text {in }}-s(t)\right]-\mu(s(t)) x(t) \\
\dot{x}(t)=[\mu(s(t))-D] x(t)
\end{array}\right.
$$

(where we used the standard technique of scaling the species level $x(t)$ in order to eliminate the constant yield) but see below for generalizations where the growth function $\mu$ can be uncertain. The states $x$ and $s$ are positive valued (and represent the species and substrate levels, respectively), the substrate input concentration $s_{\text {in }}>0$ is a constant, the dilution rate $D$ is a positive valued control that we will specify, and the growth function $\mu$ satisfies:

Assumption 1 The function $\mu$ is of class $C^{1}$ and $\mu(0)=$ 0 . Also, there is a constant $s_{M}>0$ such that $\mu^{\prime}(s)>0$ for all $s \in\left[0, s_{M}\right)$ and $\mu^{\prime}(s) \leq 0$ for all $s \in\left[s_{M}, \infty\right)$. Finally, $\mu(s)>0$ for all $s>0$.

By $C^{1}$, we mean continuously differentiable. Assumption 1 holds for all functions of the form

$$
\mu(s)=\frac{k_{1} s}{1+k_{2} s+k_{3} s^{2}},
$$

for any constants $k_{i}>0$ for $i=1$ to 3 , with $s_{M}=1 / \sqrt{k_{3}}$. Functions of the form (2) are called Haldane functions. In Fig. 1, we plot the special case of (2) and $s_{\text {in }}$ where

$$
\mu(s)=\frac{0.5 s}{1+0.25 s+2 s^{2}} \text { and } s_{\text {in }}=1
$$

including the maximizer $s_{M}=1 / \sqrt{2}$. In Appendix A, we

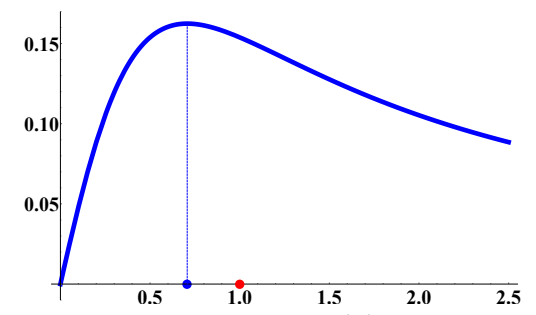

Fig. 1. Uptake Function from (3), Showing Maximizer $s_{M}=1 / \sqrt{2}$ as Blue Dot and $s_{\text {in }}=1$ as Red Dot.

prove the next lemma, where a function $\alpha:[0, \infty) \rightarrow[0, \infty)$ is defined to be of class $\mathcal{K}_{\infty}$ provided $\alpha(0)=0$ and $\alpha$ is continuous, strictly increasing, and unbounded; and $\mu_{1}^{\prime}(0)$ is the derivative from the right.

Lemma 1 If Assumption 1 holds, then we can construct a function $\mu_{1} \in C^{1} \cap \mathcal{K}_{\infty}$ and a nondecreasing $C^{1}$ function $\gamma: \mathbb{R} \rightarrow[0, \infty)$ such that $\gamma(m)=0$ for all $m \leq 0$,

$$
\mu(s)=\frac{\mu_{1}(s)}{1+\gamma(s)} \text { for all } s \geq 0
$$

$\mu_{1}^{\prime}(s)>0$ for all $s \geq 0$, and $\gamma^{\prime}(s)>0$ for all $s \geq s_{M}$.

Remark 1 If $\mu^{\prime}(s)<0$ for all $s>s_{M}$ (which holds for (2)), and $s_{\mathrm{in}}>s_{M}$, and the dilution rate $D$ is a constant $D \in\left(\mu\left(s_{\mathrm{in}}\right), \mu\left(s_{M}\right)\right) \subseteq(0, \infty)$, then the system (1) has a locally unstable positive equilibrium point of the form $\left(s_{*}, s_{\mathrm{in}}-s_{*}\right)$ and the locally stable equilibrium $\left(s_{\mathrm{in}}, 0\right)$, where $s_{*} \in\left(s_{M}, s_{\text {in }}\right)$ and $D=\mu\left(s_{*}\right)$. Our work Mazenc et al. (2013a) globally stabilized an equilibrium that can be locally exponentially stabilized by a constant dilution rate.

To explain our sampling control goals, fix any two constants $\epsilon_{1}>0$ and $\epsilon_{2}>0$ such that $\epsilon_{2}>\epsilon_{1}$, and let $\left\{t_{i}\right\}$ be a sequence of real numbers such that $0<\epsilon_{1} \leq t_{i+1}-t_{i} \leq \epsilon_{2}$ for all $i \in \mathbb{N} \cup\{0\}$, where $t_{0}=0$ and $\mathbb{N}=\{1,2, \ldots\}$. Given any constant $\tau_{f} \geq 0$, we define the function $\tau$ as follows:

$$
\tau(t)= \begin{cases}\tau_{f}, & t \in\left[0, \tau_{f}\right) \\ \tau_{f}+t-t_{j}, t & \in\left[t_{j}+\tau_{f}, t_{j+1}+\tau_{f}\right) \text { and } j \geq 0\end{cases}
$$


This is reminiscent of the representation of sampling in Fridman et al. (2004). For all $j \geq 0$ and $t \in\left[t_{j}+\tau_{f}, t_{j+1}+\right.$ $\left.\tau_{f}\right)$, we have $t-\tau(t)=t-\left(\tau_{f}+t-t_{j}\right)=t_{j}-\tau_{f}$, so $t-\tau(t)$ is piecewise constant. In the special case where $\tau_{f}=0$, we also have $t-\tau(t)=t_{j}$ for all $t \in\left[t_{j}, t_{j+1}\right)$ and $j \geq 0$.

Moreover, for all $t \geq 0$, we have

$$
0 \leq \tau(t) \leq \tau_{M}, \text { where } \tau_{M}=2 \tau_{f}+\epsilon_{2} .
$$

We assume that $s(t-\tau(t))$ is the only available measurement. Our control $D$ will be computed in terms of the delayed sampled values $s(t-\tau(t))$ of the substrate, so when $\tau_{f}=0$, the control values will be computed from the sequence of observations $\left\{s\left(t_{j}\right)\right\}$ at the sample times; see (12). When $\mu$ is known, our goal is asymptotic stabilization of $E_{*}=\left(s_{*}, s_{\text {in }}-s_{*}\right)$ for any constant $s_{*} \in\left(0, s_{\text {in }}\right)$, using our positive valued dilution rate feedback. Then the components of $E_{*}$ are positive, and $E_{*}$ is an equilibrium of (1) if and only if $D$ takes the value $\mu\left(s_{*}\right)$ when $s=s_{*}$.

\section{Main Result}

Let Assumption 1 hold with $s_{\text {in }} \geq s_{M}$, and fix any constant $s_{*} \in\left(0, s_{\text {in }}\right)$ and any functions $\gamma$ and $\mu_{1}$ that satisfy the requirements from Lemma 1 . We use the constants

$$
\begin{gathered}
\mu_{a}=\mu_{1}\left(s_{*}\right) s_{\text {in }}, \\
\varpi_{s}=\inf _{s \in\left[0, s_{\text {in }}\right]} \mu_{1}^{\prime}(s), \varpi_{l}=\sup _{s \in\left[0, s_{\text {in }}\right]} \mu_{1}^{\prime}(s), \\
\rho_{l}=\sup _{s \in\left[0, s_{\text {in }}\right]} \gamma^{\prime}(s),
\end{gathered}
$$

and

$$
\rho_{m}=\frac{\rho_{l}^{2}}{2 \varpi_{s}} \max _{l \in\left[0, s_{\mathrm{in}}\right]} \frac{\mu_{1}^{2}\left(l+1.1 \mu_{a} \tau_{M}\right)}{1+\gamma(l)}
$$

where $\tau_{M}$ satisfies (5). The preceding constants are all positive, by the properties of $\mu_{1}$ and $\gamma$.

To model uncertainties, we study the more general model

$$
\left\{\begin{array}{l}
\dot{s}(t)=D\left[s_{\text {in }}-s(t)\right]-(1+\delta(t)) \mu(s(t)) x(t) \\
\dot{x}(t)=[(1+\delta(t)) \mu(s(t))-D] x(t)
\end{array}\right.
$$

where the unknown measurable essentially bounded function $\delta:[0, \infty) \rightarrow[d, \infty)$ admits a known constant lower bound $\underline{d} \in(-1,0]$. We also assume that $\underline{d}$ and $\tau_{M}$ satisfy:

Assumption 2 The constants $\tau_{M}$ and $\underline{d}$ are such that

$$
\begin{gathered}
\frac{(1+\underline{d}) \mu_{1}\left(s_{\mathrm{in}}\right)}{1+\gamma\left(s_{\mathrm{in}}\right)}-\frac{\mu_{1}\left(s_{*}\right)}{1+\gamma\left(s_{\mathrm{in}}-\mu_{a} \tau_{M}\right)}>0, \\
\tau_{M}<\frac{1}{2 \sqrt{2 \rho_{m} \varpi_{l}} s_{\mathrm{in}}}, \text { and } \tau_{M}<\frac{1}{2 \rho_{l} s_{\text {in }} \mu_{1}\left(s_{\mathrm{in}}\right)}
\end{gathered}
$$

are all satisfied.

Note for later use that since $\gamma$ is nondecreasing, (10) gives $(1+\underline{d}) \mu_{1}\left(s_{\text {in }}\right) \geq \mu_{1}\left(s_{*}\right)$. In Fig. 2 , we illustrate how the $\delta$ 's in (9) appropriately address the uncertainty in the uptake function $\mu$, without imposing any upper bounds on the range of $\delta$. For simplicity, we took constant $\delta$ 's in Fig. 2 , but another valuable feature of $(9)$ is that it allows time varying $\delta$ 's, without any monotonicity or nonnegativity requirements on the $\delta$ 's.

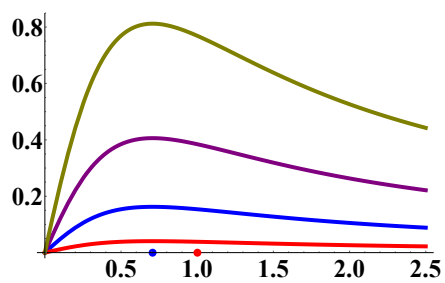

Fig. 2. Plots of $(1+\delta) \mu(s)$ with $\mu$ from $(3), s_{M}=1 / \sqrt{2}$ as Blue Dot, and $s_{\text {in }}=1$ as Red Dot, in Special Cases where $\delta=-0.75$ (Red), $\delta=0$ (Blue), $\delta=1.5$ (Purple), and $\delta=4$ (Olive).

Since $\mu_{1}$ strictly increases and $s_{*}<s_{\text {in }}$, we have $\mu_{1}\left(s_{\text {in }}\right)>$ $\mu_{1}\left(s_{*}\right)$. Therefore, it is usually easy to determine constants $d_{*} \in(-1,0)$ and $\bar{\tau}>0$ such that (10)-(11) hold for all $\underline{d} \in\left(d_{*}, 0\right]$ and $\tau_{M} \in(0, \bar{\tau})$; see our illustration in Section 5. We say that (9) is input-to-state stable (or ISS) with respect to $(\mathcal{D}, \mathcal{E}, \mathcal{S})$ for sets $\mathcal{D} \subseteq \mathbb{R}$ and $\mathcal{S} \subseteq \mathbb{R}^{2}$ and the point $\mathcal{E}=\left(s_{*}, s_{\text {in }}-s_{*}\right)$ provided that there are functions $\bar{\beta} \in \mathcal{K} \mathcal{L}$ and $\bar{\gamma} \in \mathcal{K}_{\infty}$ such that for all solutions $(s(t), x(t))$ of $(9)$ whose initial functions are valued in $\mathcal{S}$, and for all choices of $\delta:[0, \infty) \rightarrow \mathcal{D}$, we have $|(s(t), x(t))|_{\mathcal{E}} \leq \bar{\beta}\left(|(s, x)|_{\mathcal{E},\left[-\tau_{M}, 0\right]}, t\right)+\bar{\gamma}\left(|\delta|_{[0, t]}\right)$ for all $t \geq 0$, where $|(s(t), x(t))|_{\mathcal{E}}=|(s(t), x(t))-\mathcal{E}|$ is the distance to the equilibrium $\mathcal{E},|\cdot|_{[0, t]}$ is the essential supremum over $[0, t]$, $|(s, x)|_{\mathcal{E},\left[-\tau_{M}, 0\right]}$ is the essential supremum of $|(s(t), x(t))|_{\mathcal{E}}$ over $\left[-\tau_{M}, 0\right], \mathcal{K}_{\infty}$ was defined in the preceding section, and $\mathcal{K} \mathcal{L}$ is the set of all continuous functions $\bar{\beta}:[0, \infty) \times$ $[0, \infty) \rightarrow[0, \infty)$ such that (i) for each $t \geq 0$, the function $f(s)=\bar{\beta}(s, t)$ is of class $\mathcal{K}_{\infty}$ and (ii) for each $s \geq 0$, the function $g(t)=\bar{\beta}(s, t)$ is nonincreasing and satisfies $\lim _{t \rightarrow \infty} g(t)=0$. In our theorem, we assume that the initial functions are constant valued, but see Remark 3 for more general cases. Our theorem is:

Theorem 1 If Assumption 1 holds, and if $s_{\text {in }} \geq s_{M}, s_{*} \in$ $\left(0, s_{\text {in }}\right)$, and $\underline{d} \in(-1,0]$ are any constants such that $A s$ sumption 2 holds, then for any functions $\mu_{1}$ and $\gamma$ that satisfy the requirements of Lemma 1 and all constants $\bar{s} \geq s_{\text {in }}$, $\underline{x}>0$, and $\bar{d} \geq 0$, the system (9), in closed loop with

$$
D(t)=\frac{\mu_{1}\left(s_{*}\right)}{1+\gamma(s(t-\tau(t)))},
$$

is ISS with respect to $\left([-\underline{d}, \bar{d}],\left(s_{*}, s_{\mathrm{in}}-s_{*}\right),(0, \bar{s}) \times(\underline{x}, \infty)\right)$.

Remark 2 When $\delta=0$, Theorem 1 implies that $\lim _{t \rightarrow \infty}(s(t), x(t))=\left(s_{*}, s_{\text {in }}-s_{*}\right)$ for all initial conditions that are valued in $(0, \infty)^{2}$. Our proof in the next section will show that $(s(t), x(t)) \in(0, \infty)^{2}$ for all $t \geq 0$ for all initial states in $(0, \infty)^{2}$. The functions $\bar{\beta}$ and $\bar{\gamma}$ in the ISS estimate will depend on $\bar{d}, \underline{x}$, and $\bar{s}$. Our proof of Theorem 1 can be used to provide an algorithm for constructing $\bar{\beta}$ and $\bar{\gamma}$.

\section{Proof of Theorem 1}

Fix any initial state $(s(0), x(0)) \in(0, \bar{s}) \times(\underline{x}, \infty)$ and any corresponding solution $(s(t), x(t))$ for the perturbed system $(9)$, in closed loop with (12). 
First Step. We first prove that the solution $(s(t), x(t))$ is valued in $(0, \bar{s}) \times(0, \infty)$ for all $t \geq 0$. To show that $s$ and $x$ stay positive, note that at any possible time $t$ when $s(t)=0$, we would have $\dot{s}(t)>0$, since $D(t) s_{\text {in }}>0$. Hence, $(s(t), x(t)) \in(0, \infty)^{2}$ for all $t \geq 0$. Also, $\dot{s}(t)<0$ for all $t$ such that $s(t) \geq s_{\text {in }}$, so $s(t)$ stays in $(0, \bar{s})$.

We next compute useful bounds using the new variable

$$
z(t)=s_{\text {in }}-s(t)-x(t) .
$$

By summing the equations in $(9)$, we get $\dot{s}(t)+\dot{x}(t)=$ $D\left(s_{\text {in }}-s(t)-x(t)\right)=D z(t)$, so our formula (12) for $D$ and (13) combine to give

$$
\dot{z}(t)=-D(t) z(t)=-\frac{\mu_{1}\left(s_{*}\right)}{1+\gamma(s(t-\tau(t)))} z(t) .
$$

Here and in the sequel, all equalities and inequalities are along all solutions of (9), unless otherwise noted. Hence, the subadditivity of the square root gives

$$
|(x(t), s(t))| \leq s(t)+x(t)=-z(t)+s_{\text {in }} \leq|z(0)|+s_{\text {in }},
$$

since (14) implies that $|z(t)| \leq|z(0)|$. Also, since $\gamma$ is nondecreasing and $s(t)$ is bounded above by $\bar{s}$, we can integrate (14) on $[0, t]$ to get

$$
\begin{aligned}
|z(t)| & \leq|z(0)| \exp \left(-\int_{0}^{t} \frac{\mu_{1}\left(s_{*}\right) \mathrm{d} \ell}{1+\gamma(s(\ell-\tau(\ell)))}\right) \\
& \leq|z(0)| e^{\frac{-t \mu_{1}\left(s_{*}\right)}{1+\gamma(\bar{s})}} .
\end{aligned}
$$

We also use the error variable $X$ that is defined by

$$
X(t)=\left(s(t)-s_{*}, x(t)-s_{\text {in }}+s_{*}\right) .
$$

Then for all $t \geq 0$, the triangle inequality and (17) give

$$
\begin{aligned}
& |z(t)|=\left|s_{*}-s(t)-\left(x(t)-s_{\text {in }}+s_{*}\right)\right| \\
& \leq\left|s_{*}-s(t)\right|+\left|x(t)-s_{\text {in }}+s_{*}\right| \leq 2|X(t)| .
\end{aligned}
$$

Second Step. We build a function $\mathcal{T}_{a} \in \mathcal{K}_{\infty}$ such that $s(t) \in$ $\left(0, s_{\text {in }}\right)$ for all $t \geq \mathcal{T}_{a}(|X(0)|)$ for all possible values of $X(0)$. Fix an unbounded function $\tau_{*}:(0, \infty) \rightarrow[0, \infty)$ such that for each function $p:[0, \infty) \rightarrow(0, \infty)$ that satisfies the differential inequality

$$
\dot{p}(t) \geq \frac{(1+\underline{d}) \mu_{1}\left(s_{\text {in }}\right)-\mu_{1}\left(s_{*}\right)}{1+\gamma(\bar{s})} p(t) \text { for all } t \geq 0,
$$

we have $p(t) \geq 2\left(|X(0)|+s_{\text {in }}\right)$ for all $t \geq \tau_{*}(|X(0)|+p(0))$, and such that $\tau_{*}$ is strictly increasing on $\left[\left(s_{\text {in }}-s_{*}\right) / 2, \infty\right)$. Such a function $\tau_{*}$ exists because (10) implies that the numerator in (19) is positive. We next prove the following claim, by arguing by contradiction: Claim 1: $s(t)<s_{\text {in }}$ for some $t \in\left[0, \tau_{*}(|X(0)|+x(0))\right]$. Suppose that $s(t) \geq s_{\text {in }}$ for all $t \in\left[0, \tau_{*}(|X(0)|+x(0))\right]$. Then for all $t$ in this interval, the first equation in $(9)$ gives $\dot{s}(t) \leq 0$, so

$$
\begin{aligned}
& \frac{(1+\delta(t)) \mu_{1}(s(t))}{1+\gamma(s(t))}-\frac{\mu_{1}\left(s_{*}\right)}{1+\gamma(s(t-\tau(t)))} \\
& \geq \frac{(1+\delta(t)) \mu_{1}\left(s_{\text {in }}\right)}{1+\gamma(s(t))}-\frac{\mu_{1}\left(s_{*}\right)}{1+\gamma(s(t-\tau(t)))} \\
& \geq \frac{(1+\delta(t)) \mu_{1}\left(s_{\text {in }}\right)-\mu_{1}\left(s_{*}\right)}{1+\gamma(s(t-\tau(t)))} \geq \frac{(1+\underline{d}) \mu_{1}\left(s_{\text {in }}\right)-\mu_{1}\left(s_{*}\right)}{1+\gamma(\bar{s})},
\end{aligned}
$$

since $\mu_{1}$ is nondecreasing and $\delta(t) \geq \underline{d}$, where we also used the fact that $\gamma(s(t)) \leq \gamma(s(t-\tau(t))) \leq \gamma(\bar{s})$, which fol- lows because $\gamma$ is nondecreasing and $s$ is nonincreasing. Hence, by our choice (12) of $D$, the $x(t)$ component of (9) is a positive valued solution of (19), so (18) gives $x(t) \geq$ $2\left(|X(0)|+s_{\text {in }}\right) \geq|z(0)|+2 s_{\text {in }}$ at $t=\tau_{*}(|X(0)|+x(0))$, contradicting (15), since $s_{\text {in }}>0$.

Also, if $s(t)=s_{\text {in }}$, then $\dot{s}(t)<0$, so $s(t)$ cannot increase to above $s_{\text {in }}$, so $s(t)<s_{\text {in }}$ for all $t \geq \tau_{*}(|X(0)|+x(0))$. If $|X(0)| \leq \frac{1}{2}\left(s_{\text {in }}-s_{*}\right)$, then $\left|s(0)-s_{*}\right| \leq \frac{1}{2}\left(s_{\text {in }}-s_{*}\right)$, which gives $s(0)-s_{\text {in }}=s(0)-s_{*}-\left(s_{\text {in }}-s_{*}\right) \leq-\frac{1}{2}\left(s_{\text {in }}-s_{*}\right)<0$, so $s(t)$ stays in $\left(0, s_{\text {in }}\right)$. Also, $(15)$ and $(18)$ give $0<x(0) \leq$ $|z(0)|+s_{\text {in }} \leq 2|X(0)|+s_{\text {in }}$, which gives $\tau_{*}\left(3|X(0)|+s_{\text {in }}\right) \geq$ $\tau_{*}(|X(0)|+x(0))$ if $|X(0)| \geq\left(s_{\text {in }}-s_{*}\right) / 2$. Therefore,

$$
\mathcal{T}_{a}(p)= \begin{cases}\frac{2 p}{s_{\mathrm{in}}-s_{*}} \tau_{*}\left(\frac{3\left(s_{\mathrm{in}}-s_{*}\right)}{2}+s_{\mathrm{in}}\right), & p<\frac{s_{\text {in }}-s_{*}}{2} \\ \tau_{*}\left(3 p+s_{\mathrm{in}}\right), & p \geq \frac{s_{\text {in }}-s_{*}}{2}\end{cases}
$$

satisfies our requirements. (The formula for the restriction of $\mathcal{T}_{a} \in \mathcal{K}_{\infty}$ to $\left[0,\left(s_{\text {in }}-s_{*}\right) / 2\right)$ was chosen to ensure that $\mathcal{T}_{a}$ is 0 at 0 , strictly increasing on $\left[0,\left(s_{\text {in }}-s_{*}\right) / 2\right)$, and continuous at $\left(s_{\text {in }}-s_{*}\right) / 2$, which are requirements for $\mathcal{K}_{\infty}$ functions. The validity of the formula for $\mathcal{T}_{a}$ on $\left[\left(s_{\mathrm{in}}-\right.\right.$ $\left.\left.s_{*}\right) / 2, \infty\right)$ follows from Claim 1.)

Third Step. We build a function $\mathcal{T}_{c} \in \mathcal{K}_{\infty}$ and a constant $s_{\triangle} \in\left(0, s_{\text {in }}\right)$ such that $s(t)<s_{\triangle}$ for all $t \geq \mathcal{T}_{c}(|X(0)|)$, where $s \triangle$ will be independent of $X(0)$ and $\delta$. First, notice that our choice (13) of $z$ implies that

$$
\begin{aligned}
\dot{x}(t)= & {\left[\frac{(1+\delta(t)) \mu_{1}\left(s_{\mathrm{in}}-x(t)-z(t)\right)}{1+\gamma\left(s_{\mathrm{in}}-x(t)-z(t)\right)}\right.} \\
& \left.-\frac{\mu_{1}\left(s_{*}\right)}{1+\gamma\left(s_{\mathrm{in}}-x(t)-z(t)+s(t-\tau(t))-s(t)\right)}\right] x(t) .
\end{aligned}
$$

Also, since the initial functions for $s$ are constant, we can use the Fundamental Theorem of Calculus to get

$$
\begin{aligned}
& s(t-\tau(t))-s(t)=-\int_{t_{d}}^{t} \dot{s}(m) \mathrm{d} m \text { and } \\
& \int_{t_{d}}^{t} \dot{s}(m) \mathrm{d} m=\int_{t_{d}}^{t}\left\{\left(\frac{\mu_{1}\left(s_{*}\right)}{1+\gamma(s(m-\tau(m)))}\right.\right. \\
& \left.\left.-\frac{(1+\delta(m)) \mu_{1}(s(m))}{1+\gamma(s(m))}\right)\left[s_{\text {in }}-s(m)\right]\right\} \mathrm{d} m \\
& +\int_{t_{d}}^{t} \frac{(1+\delta(m)) \mu_{1}(s(m))}{1+\gamma(s(m))} z(m) \mathrm{d} m
\end{aligned}
$$

for all $t \geq 0$, where $t_{d}=\max \{0, t-\tau(t)\}$. Since $s_{\text {in }}-s(t) \geq$ 0 and $1+\delta(t) \geq 1+\underline{d}>0$ hold for all $t \geq \mathcal{T}_{a}(|X(0)|)$, we get the following for all $t \geq \mathcal{T}_{a}(|X(0)|)$ :

$$
\begin{aligned}
& s(t-\tau(t))-s(t)=-\int_{t_{d}}^{t} \dot{s}(m) \mathrm{d} m \geq \\
& -\mu_{a} \tau_{M}-\left\{(1+\bar{d}) \mu_{1}\left(s_{\mathrm{in}}\right) \int_{t_{d}}^{t}|z(m)| \mathrm{d} m\right\},
\end{aligned}
$$

by combining (22)-(23), where $\mu_{a}$ and $\tau_{M}$ are from (5) and (6), since the quantity in curly braces in (23) is bounded above by $\mu_{1}\left(s_{*}\right)\left(s_{\text {in }}-s(m)\right)$ and $\gamma$ is nonnegative valued.

Since $\gamma$ is nondecreasing, we can use (24) to obtain

$$
\begin{aligned}
& -\frac{\mu_{1}\left(s_{*}\right)}{1+\gamma\left(s_{\mathrm{in}}-x(t)-z(t)+s(t-\tau(t))-s(t)\right)} \geq \\
& -\frac{\mu_{1}\left(s_{*}\right)}{1+\gamma\left(s_{\text {in }}-x(t)-\mu_{a} \tau_{M}-z(t)-(1+\bar{d}) \mu_{1}\left(s_{\text {in }}\right) \int_{t_{d}}^{t}|z(m)| \mathrm{d} m\right)} .
\end{aligned}
$$

Using (25) to lower bound the second term inside the brackets in (21), and then the nonnegativity of $x(t)$, we get $\dot{x}(t) \geq$ 
$\left[q+\kappa(x(t))+\lambda\left(x(t), z_{t}\right)\right] x(t)$ for all $t \geq \mathcal{T}_{a}(|X(0)|)$, where $q$ denotes the left side of (10),

$$
\begin{aligned}
& \lambda\left(x(t), z_{t}\right)=\frac{(1+\underline{d}) \mu_{1}\left(s_{\mathrm{in}}-x(t)-z(t)\right)}{1+\gamma\left(s_{\mathrm{in}}-x(t)-z(t)\right)}-\frac{(1+\underline{d}) \mu_{1}\left(s_{\mathrm{in}}-x(t)\right)}{1+\gamma\left(s_{\mathrm{in}}-x(t)\right)}+ \\
& \frac{\mu_{1}\left(s_{*}\right)}{1+\gamma\left(s_{\mathrm{in}}-x(t)-\mu_{a} \tau_{M}\right)}- \\
& \frac{\mu_{1}\left(s_{*}\right)}{1+\gamma\left(s_{\mathrm{in}}-x(t)-\mu_{a} \tau_{M}-z(t)-(1+\bar{d}) \mu_{1}\left(s_{\mathrm{in}}\right) \int_{t_{d}}^{t}|z(m)| \mathrm{d} m\right)} \\
& \text { and } \kappa(x)=\frac{(1+\underline{d}) \mu_{1}\left(s_{\mathrm{in}}-x\right)}{1+\gamma\left(s_{\mathrm{in}}-x\right)}-\frac{(1+\underline{d}) \mu_{1}\left(s_{\mathrm{in}}\right)}{1+\gamma\left(s_{\mathrm{in}}\right)} \\
& \quad+\frac{\mu_{1}\left(s_{*}\right)}{1+\gamma\left(s_{\mathrm{in}}-\mu_{a} \tau_{M}\right)}-\frac{\mu_{1}\left(s_{*}\right)}{1+\gamma\left(s_{\mathrm{in}}-x-\mu_{a} \tau_{M}\right)},
\end{aligned}
$$

and $z_{t}$ is defined by $z_{t}(\ell)=z(t+\ell)$ for all $\ell \geq 0$, and where we extend the domain of $\mu_{1}$ so that $\mu_{1}$ is zero on $(-\infty, 0)$.

We can also use (15) and our exponential decay estimate (16) on $z(t)$ to find a function $\mathcal{T}_{b} \in \mathcal{K}_{\infty}$ such that $\lambda\left(x(t), z_{t}\right) \geq-q / 2$ and therefore also

$$
\dot{x}(t) \geq[0.5 q+\kappa(x(t))] x(t)
$$

for all $t \geq \mathcal{T}_{b}(|X(0)|)$. Also, since $\kappa(0)=0$, the continuity of $\kappa$ provides a constant $x_{p} \in\left(0,\left(s_{\text {in }}-s_{*}\right) / 2\right)$ such that for all $x \in\left[0, x_{p}\right]$, we have $\kappa(x) \geq-0.25 q$. By enlarging $\mathcal{T}_{b}$, we can also assume that for all $t \geq \mathcal{T}_{b}(|X(0)|)$, we have

$$
x(t) \geq x_{p} .
$$

This follows because if $t \geq \mathcal{T}_{b}(|X(0)|)$, then (26) gives

$$
\dot{x}(t) \geq[0.5 q+\kappa(x(t))] x(t) \geq \frac{q}{4} x(t) \text { if } x(t) \in\left(0, x_{p}\right] .
$$

To enlarge $\mathcal{T}_{b}$, notice that if $|X(0)| \leq \frac{1}{2}\left(s_{\text {in }}-s_{*}\right)$, then our formula (17) for $X$ gives $\left|x(0)-\left(s_{\text {in }}-s_{*}\right)\right| \leq 0.5\left(s_{\text {in }}-s_{*}\right)$, and then the triangle inequality gives $x(0) \geq 0.5\left(s_{\text {in }}-s_{*}\right) \geq$ $x_{p}$, which gives $x(t) \geq x_{p}$ for all $t \geq 0$, by (28). On the other hand, we can use (28) to find a positive valued function $\mathcal{M}$ such that $x(t) \geq x_{p}$ for all $t \geq \mathcal{M}(x(0))$ and all choices of $x(0) \geq \underline{x}$ and such that $\mathcal{M}$ is strictly increasing on $[\underline{x}, \infty)$. Also, the triangle inequality gives

$|X(0)|+s_{\text {in }}-s_{*} \geq\left|x(0)-\left(s_{\text {in }}-s_{*}\right)\right|+s_{\text {in }}-s_{*} \geq x(0)$.

Hence, $\mathcal{M}\left(|X(0)|+s_{\text {in }}-s_{*}\right) \geq \mathcal{M}(x(0))$ if $|X(0)| \geq$ $0.5\left(s_{\text {in }}-s_{*}\right)$, so it suffices to enlarge $\mathcal{T}_{b}$ so that

$$
\mathcal{T}_{b}(r) \geq \begin{cases}\frac{2 r}{s_{\text {in }}-s_{*}} \mathcal{M}\left(1.5\left(s_{\text {in }}-s_{*}\right)\right), & r \leq \frac{s_{\text {in }}-s_{*}}{2} \\ \mathcal{M}\left(r+s_{\text {in }}-s_{*}\right), & r>\frac{s_{\text {in }}-s_{*}}{2}\end{cases}
$$

where the formula for the lower bound for $\mathcal{T}_{b}$ on $\left[0,\left(s_{\text {in }}-\right.\right.$ $\left.s_{*}\right) / 2$ ] was chosen for the same reason that we chose the corresponding part of the formula for $\mathcal{T}_{a}$ in the second step.

Also, our exponential decay condition (16) on $z(t)$ and (18) provide a function $\mathcal{M}_{*} \in \mathcal{K}_{\infty}$ such that for all $t \geq$ $\mathcal{M}_{*}(|X(0)|)$, the inequalities

$$
|z(t)| \leq 0.25 x_{p} \leq 0.25 s_{\text {in }}
$$

hold. Using the definition of $z$ and (27)-(30), and setting $s_{\triangle}=s_{\text {in }}-0.75 x_{p}$, we deduce that $s_{\triangle} \in\left(0, s_{\text {in }}\right)$ and

$$
\begin{aligned}
& s(t)=-x(t)-z(t)+s_{\text {in }} \leq-x(t)+0.25 x_{p}+s_{\text {in }} \\
& \leq-x_{p}+0.25 x_{p}+s_{\text {in }}=s_{\triangle}
\end{aligned}
$$

for all $t \geq \mathcal{T}_{c}(|X(0)|)$, where $\mathcal{T}_{c}=\max \left\{\mathcal{T}_{a}, \mathcal{T}_{b}, \mathcal{M}_{*}\right\} \in \mathcal{K}_{\infty}$.

Fourth Step. We construct a functional $\mathcal{U}_{1}$, which we later add to a double integral term to prove our ISS property. Using $z$ as defined in (13), and the structure of $\mu$ in (4), we get this for all $t \geq 0$ :

$$
\begin{aligned}
& \dot{s}(t)=\left[\frac{\mu_{1}\left(s_{*}\right)}{1+\gamma(s(t-\tau(t)))}-\frac{\mu_{1}(s(t))}{1+\gamma(s(t))}\right]\left[s_{\text {in }}-s(t)\right] \\
& +(1+\delta(t)) \mu(s(t)) z(t)-\delta(t) \mu(s(t))\left(s_{\text {in }}-s(t)\right) .
\end{aligned}
$$

Since the Fundamental Theorem of Calculus gives

$$
\gamma(s(t))-\gamma(s(t-\tau))=\int_{t-\tau(t)}^{t} \gamma^{\prime}(s(m)) \dot{s}(m) \mathrm{d} m,
$$

we can use a common denominator in (32) to get

$$
\begin{aligned}
& \dot{s}(t)= \\
& \left\{\frac{\left[\mu_{1}\left(s_{*}\right)-\mu_{1}(s(t))\right][1+\gamma(s(t))]+\mu_{1}(s(t)) \mathcal{I}(t)}{[1+\gamma(s(t-\tau(t)))][1+\gamma(s(t))]}\left[s_{\text {in }}-s(t)\right]\right\} \\
& +\left\{(1+\delta(t)) \mu(s(t)) z(t)-\delta(t) \mu(s(t))\left(s_{\text {in }}-s(t)\right)\right\}
\end{aligned}
$$

where $\mathcal{I}(t)=\int_{t-\tau(t)}^{t} \gamma^{\prime}(s(m)) \dot{s}(m) \mathrm{d} m$.

We next choose

$$
\mathcal{U}_{1}(s)=\int_{0}^{s-s_{*}} \frac{m}{s_{\mathrm{in}}-s_{*}-m} \mathrm{~d} m
$$

which is $C^{1}$ over $\left[0, s_{\text {in }}\right)$, and nonnegative valued at $s(t)$ for all times $t \geq \mathcal{T}_{c}(|X(0)|)$, by the third step. It follows from (34) that its time derivative along all solutions of the closed loop system for all $t \geq \mathcal{T}_{c}(|X(0)|)$ satisfies

$$
\begin{aligned}
& \dot{\mathcal{U}}_{1}(t)=\frac{s(t)-s_{*}}{s_{\text {in }}-s}(1+\delta(t)) \mu(s(t)) z(t) \\
& -\delta(t) \mu(s(t))\left(s(t)-s_{*}\right) \\
& \frac{\left[\mu_{1}\left(s_{*}\right)-\mu_{1}(s(t))\right][1+\gamma(s(t))]+\mu_{1}(s(t)) \mathcal{I}(t)}{[1+\gamma(s(t-\tau(t)))][1+\gamma(s(t))]}\left(s(t)-s_{*}\right),
\end{aligned}
$$

where we use $\dot{\mathcal{U}}(t)$ to denote $(d / d t) \mathcal{U}(s(t))$ to make our notation concise. From the third step and the fact that $\mu(s) \leq$ $\mu\left(s_{M}\right)$ for all $s \geq 0$, we deduce that if $t \geq \mathcal{T}_{c}(|X(0)|)$, then

$$
\begin{aligned}
& \dot{\mathcal{U}}_{1}(t) \leq\left|s(t)-s_{*}\right| \frac{\mu_{1}(s(t)) \int_{t-\tau(t)}^{t} \gamma^{\prime}(s(m))|\dot{s}(m)| \mathrm{d} m}{1+\gamma(s(t-\tau(t)))} \\
& +\frac{\left(s(t)-s_{*}\right)\left(\mu_{1}\left(s_{*}\right)-\mu_{1}(s(t))\right)}{1+\gamma(s(t-\tau(t)))}+\bar{c}\left|s(t)-s_{*}\right|(|z(t)|+|\delta(t)|)
\end{aligned}
$$

holds, where $\bar{c}=\mu\left(s_{M}\right) \max \left\{(1+\bar{d}) /\left(s_{\text {in }}-s_{\triangle}\right), 1\right\}$.

From the definition of $\rho_{l}$ in $(7)$ and the fact that $s(t)<s_{\Delta}$ holds for all $t \geq \mathcal{T}_{c}(|X(0)|)$, we obtain

$$
\begin{aligned}
\dot{\mathcal{U}}_{1}(t) \leq & {\left[\frac{\left(s(t)-s_{*}\right)\left(\mu_{1}\left(s_{*}\right)-\mu_{1}(s(t))\right)}{1+\gamma(s(t-\tau(t))}\right]+\hat{c}(t) } \\
& +\rho_{l}\left|s(t)-s_{*}\right| \frac{\mu_{1}(s(t)) \int_{t-\tau(t)}^{t}|\dot{s}(m)| \mathrm{d} m}{1+\gamma(s(t-\tau(t)))} \\
\leq & \frac{\left(s(t)-s_{*}\right)\left(\mu_{1}\left(s_{*}\right)-\mu_{1}(s(t))\right)}{2[1+\gamma(s(t-\tau(t)))]} \\
& -\varpi_{s} \frac{\left(s(t)-s_{*}\right)^{2}}{2[1+\gamma(s(t-\tau(t)))]}+\hat{c}(t) \\
& +\left\{\left|s(t)-s_{*}\right|\right\}\left\{\mu_{1}\left(s(t-\tau(t))+1.1 \mu_{a} \tau_{M}\right)\right. \\
& \left.\times \rho_{l} \int_{t-\tau(t)}^{t}|\dot{s}(m)| \mathrm{d} m\right\} \frac{1}{1+\gamma(s(t-\tau(t)))}
\end{aligned}
$$

for all $t \geq \mathcal{T}_{d}(|X(0)|)$, where $\hat{c}(t)=\bar{c}\left|s(t)-s_{*}\right|(|z(t)|+$ $|\delta(t)|)$ and $\mathcal{T}_{d} \in \mathcal{K}_{\infty}$ is such that $\mathcal{T}_{d}(r) \geq \mathcal{T}_{c}(r)$ for all $r \geq 0$ and is such that the term in curly braces in (24) is bounded 
above by $0.1 \mu_{a} \tau_{M}$ for all $t \geq \mathcal{T}_{d}(|X(0)|)$; such a $\mathcal{T}_{d}$ can be found using the exponential decay estimate (16) on $z(t)$ and (18), and will depend on $\bar{d}$. The second inequality in (37) follows from the definition of $\varpi_{s}$ in (7), which gives

$$
\left(\mu_{1}\left(s_{*}\right)-\mu_{1}(s(t))\left(s_{*}-s(t)\right) \geq \varpi_{s}\left(s_{*}-s(t)\right)^{2}\right.
$$

when we use the Mean Value Theorem (to upper bound the first quantity in square brackets in (37) by the first two terms after the second inequality in (37)).

Hence, our choice of $\rho_{m}>0$ from (8) gives

$$
\begin{aligned}
\dot{\mathcal{U}}_{1}(t) \leq & \frac{\left(s(t)-s_{*}\right)\left(\mu_{1}\left(s_{*}\right)-\mu_{1}(s(t))\right)}{2[1+\gamma(s(t-\tau(t)))]} \\
& +\frac{\rho_{l}^{2}}{2 \varpi_{s}} \frac{\mu_{1}^{2}\left(s(t-\tau(t))+1.1 \mu_{a} \tau_{M}\right)}{1+\gamma(s(t-\tau(t)))} \tau_{M} \\
& \times \int_{t-\tau(t)}^{t}(\dot{s}(m))^{2} \mathrm{~d} m+\hat{c}(t) \\
\leq & \frac{\left(s(t)-s_{*}\right)\left(\mu_{1}\left(s_{*}\right)-\mu_{1}(s(t))\right)}{2[1+\gamma(s(t-\tau(t)))]} \\
& +\rho_{m} \tau_{M} \int_{t-\tau(t)}^{t}(\dot{s}(m))^{2} \mathrm{~d} m+\hat{c}(t)
\end{aligned}
$$

where we used Young's inequality $a b \leq \frac{\varpi_{s}}{2} a^{2}+\frac{1}{2 \varpi_{s}} b^{2}$ with $a$ and $b$ chosen to be the first and second terms in curly braces in (37) respectively, and Jensen's inequality to get

$$
\left(\int_{t-\tau(t)}^{t}|\dot{s}(m)| \mathrm{d} m\right)^{2} \leq \tau_{M} \int_{t-\tau(t)}^{t}(\dot{s}(m))^{2} \mathrm{~d} m
$$

Fifth Step. We define

$$
\mathcal{U}_{2}\left(s_{t}\right)=\mathcal{U}_{1}(s(t))+2 \rho_{m} \tau_{M} \int_{t-\tau_{M}}^{t} \int_{\ell}^{t}(\dot{s}(m))^{2} \mathrm{~d} m \mathrm{~d} \ell .
$$

along all solutions of our dynamics. Then (39) gives

$$
\begin{aligned}
& \dot{\mathcal{U}}_{2}(t) \leq \frac{\left(s(t)-s_{*}\right)\left(\mu_{1}\left(s_{*}\right)-\mu_{1}(s(t))\right)}{2[1+\gamma(s(t-\tau(t)))]} \\
& -\rho_{m} \tau_{M} \int_{t-\tau_{M}}^{t}(\dot{s}(m))^{2} \mathrm{~d} m+2 \rho_{m} \tau_{M}^{2}(\dot{s}(t))^{2}+\hat{c}(t),
\end{aligned}
$$

for all $t \geq \mathcal{T}_{d}(|X(0)|)$. Let $a$ and $b$ denote the first and second terms in curly braces in (34) respectively, and let $p_{0} \in(0,1)$ be a constant that we will specify later. Then (34) gives $(\dot{s}(t))^{2} \leq\left(1+p_{0}\right) a^{2}+\left(1+1 / p_{0}\right) b^{2}$, so (42) gives

$$
\begin{aligned}
& \dot{\mathcal{U}}_{2}(t) \leq \frac{\left(s(t)-s_{*}\right)\left(\mu_{1}\left(s_{*}\right)-\mu_{1}(s(t))\right)}{2[1+\gamma(s(t-\tau(t)))]}-\rho_{m} \tau_{M} \int_{t-\tau_{M}}^{t}(\dot{s}(m))^{2} \mathrm{~d} m \\
& +\left[\frac{\left[\mu_{1}\left(s_{*}\right)-\mu_{1}(s(t))\right][1+\gamma(s(t))]+\mu_{1}(s(t)) \rho_{l} \int_{t-\tau(t)}^{t}|\dot{s}(m)| \mathrm{d} m}{[1+\gamma(s(t-\tau(t)))][1+\gamma(s(t))]}\right]^{2} \\
& \quad \times 2\left(1+p_{0}\right) \rho_{m} \tau_{M}^{2}\left[s_{\text {in }}-s(t)\right]^{2}+\hat{c}(t) \\
& +4\left(1+1 / p_{0}\right) \mu^{2}\left(s_{M}\right)\left((1+\bar{d})^{2} z^{2}(t)+s_{\text {in }}^{2} \delta^{2}(t)\right) \rho_{m} \tau_{M}^{2},
\end{aligned}
$$

where we also used the relation $\left(c_{1}+c_{2}\right)^{2} \leq 2\left(c_{1}^{2}+c_{2}^{2}\right)$ for suitable nonnegative values $c_{1}$ and $c_{2}$ to bound $b^{2}$.

Using our definition of $\varpi_{l}$ in $(7),(40)$, and $(\bar{a}+\bar{b})^{2} \leq$ $2 \bar{a}^{2}+2 \bar{b}^{2}$ for suitable choices of $\bar{a} \geq 0$ and $\bar{b} \geq 0$, it follows that for all $t \geq \mathcal{T}_{d}(|X(0)|)$, we have

$$
\begin{aligned}
& \dot{\mathcal{U}}_{2}(t) \leq \hat{c}(t)+c_{* *}(t) \\
& +\frac{\left(s(t)-s_{*}\right)\left(\mu_{1}\left(s_{*}\right)-\mu_{1}(s(t))\right)}{2[1+\gamma(s(t-\tau(t)))]}-\rho_{m} \tau_{M} \int_{t-\tau_{M}}^{t}(\dot{s}(m))^{2} \mathrm{~d} m \\
& +4\left(1+p_{0}\right) \rho_{m} \tau_{M}^{2}\left[\frac{\left(\mu_{1}\left(s_{*}\right)-\mu_{1}(s(t))\right)^{2}}{[1+\gamma(s(t-\tau(t)))]^{2}}\right. \\
& \left.+\frac{\mu_{1}^{2}(s(t)) \rho_{l}^{2} \tau_{M} \int_{t-\tau(t)}^{t}(\dot{s}(m))^{2} \mathrm{~d} m}{[1+\gamma(s(t-\tau(t)))]^{2}[1+\gamma(s(t))]^{2}}\right]\left[s_{\mathrm{in}}-s(t)\right]^{2}
\end{aligned}
$$

and so also

$$
\begin{aligned}
& \dot{\mathcal{U}}_{2}(t) \leq \frac{\left(s(t)-s_{*}\right)\left(\mu_{1}\left(s_{*}\right)-\mu_{1}(s(t))\right)}{2[1+\gamma(s(t-\tau(t)))]} \\
& +4\left(1+p_{0}\right) \rho_{m} \tau_{M}^{2} \varpi_{l} \frac{\left(s_{*}-s(t)\right)\left(\mu_{1}\left(s_{*}\right)-\mu_{1}(s(t))\right)}{[1+\gamma(s(t-\tau(t)))]^{2}}\left[s_{\mathrm{in}}-s(t)\right]^{2} \\
& +\left(4 \rho_{m} \tau_{M}^{2} \frac{\mu_{1}^{2}(s(t)) \rho_{l}^{2}\left(1+p_{0}\right)}{[1+\gamma(s(t-\tau(t)))]^{2}[1+\gamma(s(t))]^{2}}\left[s_{\mathrm{in}}-s(t)\right]^{2}-\rho_{m}\right) \\
& \quad \times \tau_{M} \int_{t-\tau_{M}}^{t}(\dot{s}(m))^{2} \mathrm{~d} m+\hat{c}(t)+c_{* *}(t)
\end{aligned}
$$

where $c_{* *}(t)=c_{*}\left(z^{2}(t)+\delta^{2}(t)\right)$ and $c_{*}=4(1+$ $\left.1 / p_{0}\right) \mu^{2}\left(s_{M}\right) \max \left\{(1+\bar{d})^{2}, s_{\text {in }}^{2}\right\} \rho_{m} \tau_{M}^{2}$.

Hence, for all $t \geq \mathcal{T}_{d}(|X(0)|)$, we have

$$
\begin{aligned}
& \dot{\mathcal{U}}_{2}(t) \leq\left[-1+8 \rho_{m}\left(1+p_{0}\right) \tau_{M}^{2} \varpi_{l} \frac{\left[s_{\text {in }}-s(t)\right]^{2}}{[1+\gamma(s(t-\tau(t)))]}\right] \\
& \times \frac{\left(s_{*}-s(t)\right)\left(\mu_{1}\left(s_{*}\right)-\mu_{1}(s(t))\right)}{2[1+\gamma(s(t-\tau(t)))]} \\
& +\left(4 \tau_{M}^{2} \rho_{l}^{2} \frac{\mu_{1}^{2}(s(t))\left(1+p_{0}\right)}{[1+\gamma(s(t-\tau(t)))]^{2}}\left[s_{\text {in }}-s(t)\right]^{2}-1\right) \\
& \times \rho_{m} \tau_{M} \int_{t-\tau_{M}}^{t}(\dot{s}(m))^{2} \mathrm{~d} m+\hat{c}(t)+c_{* *}(t) \\
& \leq\left\{8\left(1+p_{0}\right) \rho_{m} \varpi_{l} s_{\text {in }}^{2} \tau_{M}^{2}-1\right\} \\
& \times \frac{\left(s_{*}-s(t)\right)\left(\mu_{1}\left(s_{*}\right)-\mu_{1}(s(t))\right)}{2[1+\gamma(s(t-\tau(t)))]} \\
& +\left\{4\left(1+p_{0}\right)\left(\rho_{l} \mu_{1}(s(t)) s_{\text {in }} \tau_{M}\right)^{2}-1\right\} \\
& \times \rho_{m} \tau_{M} \int_{t-\tau_{M}}^{t}(\dot{s}(m))^{2} \mathrm{~d} m+\hat{c}(t)+c_{* *}(t),
\end{aligned}
$$

since $\mu_{1}$ is nondecreasing. We conclude from our upper bounds from (11) on $\tau_{M}$, the fact that $\mu_{1}(s(t)) \leq \mu_{1}\left(s_{\text {in }}\right)$, the Mean Value Theorem applied to $\mu_{1}$, the bounds

$$
\begin{aligned}
& \int_{t-\tau_{M}}^{t} \int_{\ell}^{t}(\dot{s}(m))^{2} \mathrm{~d} m \mathrm{~d} \ell \leq \tau_{M} \int_{t-\tau_{M}}^{t}(\dot{s}(m))^{2} \mathrm{~d} m \\
& \text { and } \frac{1}{2 s_{\text {in }}}\left(s-s_{*}\right)^{2} \leq \mathcal{U}_{1}(s) \leq \frac{\left(s-s_{*}\right)^{2}}{2\left(s_{\mathrm{in}}-\max \left\{s_{*}, s_{\triangle}\right\}\right)},
\end{aligned}
$$

and the positivity of $\varpi_{s}$ in (38) that for a small enough constant $p_{0} \in(0,1)$, there is a constant $c_{e}>0$ such that along all trajectories of $(9)$, we have

$$
\dot{\mathcal{U}}_{2}(t) \leq-c_{e} \mathcal{U}_{2}\left(s_{t}\right)+c_{*}\left(z^{2}(t)+\delta^{2}(t)\right)+\hat{c}(t)
$$

for all $t \geq \mathcal{T}_{d}(|X(0)|)$, since the quantities in curly braces in (43) are negative. Using Young's inequality twice to upper bound $\hat{c}(t)$ and then the relation $(|z(t)|+|\delta(t)|)^{2} \leq$ $2\left(z^{2}(t)+\delta^{2}(t)\right)$ and the lower bound on $\mathcal{U}_{1}$ from $(44)$ yields positive constants $c_{f}$ and $c_{g}$ such that

$$
\dot{\mathcal{U}}_{2}(t) \leq-c_{f} \mathcal{U}_{2}\left(s_{t}\right)+c_{g}\left(z^{2}(t)+\delta^{2}(t)\right)
$$

for all $t \geq \mathcal{T}_{d}(|X(0)|)$. The theorem now follows directly from the exponential decay condition (14) on $z(t)$ and from integrating (46), using the bounds (44); see Appendix B.

Remark 3 The constantness of the initial functions was used to obtain (23). Theorem 1 remains true if instead of assuming that both components $s$ and $x$ of the initial functions are constant, we only assume that the initial function for $s$ is constant (by the same proof). In fact, we can drop the constantness assumptions on the initial functions entirely, by viewing the calculations in the proof of Theorem 1 as holding for $t \geq \tau_{M}$, instead of all $t \geq 0$. See Mazenc et al. (2016), where the case of nonconstant initial functions was handled in the special case where the $\delta$ 's are zero. 


\section{Example}

Our theorem ensures asymptotic convergence for all componentwise positive initial states. To illustrate the theorem in an example, we use the growth rate and constant

$$
\mu(s)=\frac{0.5 s}{1+0.25 s+2 s^{2}} \text { and } s_{\text {in }}=1 .
$$

Then Assumption 1 and the requirements of Lemma 1 hold using $s_{M}=1 / \sqrt{2}$ and $\mu_{1}(s)=0.5 s$ and $\gamma(s)=0.25 s+2 s^{2}$ for all $s \geq 0$.

We take $s_{*}=0.8 \in\left(s_{M}, s_{\text {in }}\right)$. Then the constants from Section 3 are $\varpi_{s}=\varpi_{l}=0.5, \rho_{l}=0.25+4=17 / 4$, $\mu_{a}=0.4$, and

$$
\rho_{m}=\left(\frac{17}{4}\right)^{2} \frac{1}{4} \max _{\ell \in[0,1]} \frac{\left(\ell+0.44 \tau_{M}\right)^{2}}{1+0.25 \ell+2 \ell^{2}} .
$$

If we set $\tau_{M}=0.5$ in (48), then we obtain $\rho_{m}=2.371$, so $\rho_{m} \leq 2.371$ for all $\tau_{M} \in(0,0.5]$. The requirements (11) from Assumption 2 hold if $\tau_{M} \in(0,4 / 17)$. Also, our condition (10) from Assumption 2 reads

$$
\begin{aligned}
& \frac{0.5(1+\underline{d})}{13 / 4}-\frac{0.5(0.8)}{1+0.25\left(1-0.4 \tau_{M}\right)+2\left(1-0.4 \tau_{M}\right)^{2}} \\
& =0.0264>0
\end{aligned}
$$

when we choose any $\tau_{M} \in(0,0.24)$ and $\underline{d}=0$. Hence, all of our assumptions hold if $\tau_{M} \leq 0.23$ and $\underline{d}=0$.

To illustrate our findings, we ran Mathematica simulations with $\tau_{M}=0.23, t_{j}=0.23 j$ for all $j \geq 0$, and $\tau_{f}=0$, using the NDSolve command and the closed loop dynamics

$$
\left\{\begin{aligned}
\dot{s}(t)= & \frac{0.4(1-s(t))}{1+0.25 s(0.23\lfloor t / 0.23\rfloor)+2 s^{2}(0.23\lfloor t / 0.23\rfloor)} \\
& -\frac{0.5(1+\delta(t)) s(t)}{1+0.25 s(t)+2 s^{2}(t)} x(t) \\
\dot{x}(t)= & \frac{0.5(1+\delta(t)) s(t) x(t)}{1+0.25 s(t)+2 s^{2}(t)} \\
& -\frac{0.4 x(t)}{1+0.25 s(0.23\lfloor t / 0.23\rfloor)+2 s^{2}(0.23\lfloor t / 0.23\rfloor)}
\end{aligned}\right.
$$

for different choices of the perturbation $\delta(t)$, where $\lfloor a\rfloor=$ $\max \{j \in\{0,1,2, \ldots\}: j \leq a\}$ is the floor function; see Mathematica (2015). In Figs. 3-4, we plot the components of the state and the control for different initial states, with our control $D(t)$ from Theorem 1. For Fig. 3, we chose $\delta=0$, so $(s(t), x(t))$ converges to $\left(s_{*}, s_{\text {in }}-s_{*}\right)=(0.8,0.2)$. In Fig. 4, we simulated our closed loop system with our feedback with the choice $\delta(t)=0.15(1+\sin (t))$, and the states instead converge toward an oscillation around the equilibrium, which agrees with our ISS result. Hence, our simulations help validate our theory. Our results also apply if we allow nonzero $\tau_{f}$ 's and nonconstant sample rates.

\section{Conclusions}

We used a new barrier Lyapunov function approach to prove input-to-state stability with respect to uncertainties in the growth functions in two state chemostats, in closed loop with output feedback controls. Our results are significant because only delayed and sampled measurements of the substrate level are available for use in the control, and because we allow a general class of growth functions that
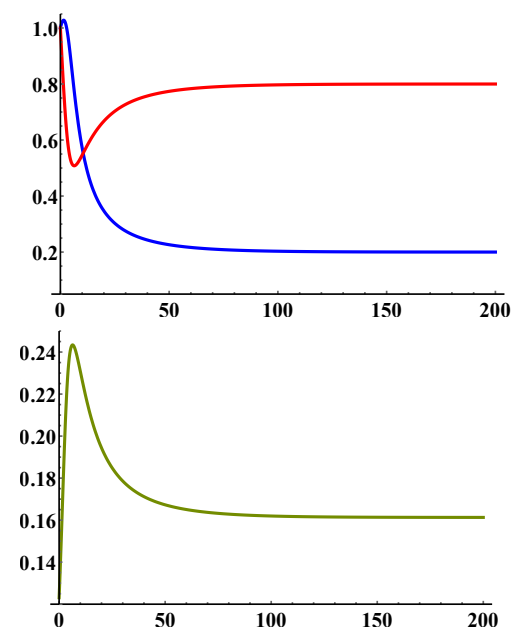

Fig. 3. Top: Solution $(s(t), x(t))$ of $(50)$ for Initial State $(1,1)$ Converging to $(0.8,0.2)$ with $\tau_{M}=0.23, \delta(t)=0, s(t)$ in Red, and $x(t)$ in Blue. Bottom: Control $D(t)$ in Green.
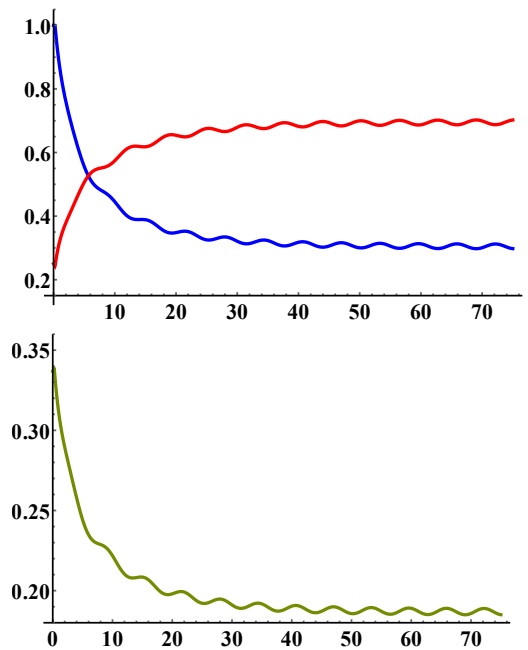

Fig. 4. Top: Solution $(s(t), x(t))$ of $(50)$ for Initial State $(0.25,1)$ with $\tau_{M}=0.23, \delta(t)=0.15(1+\sin (t)), s(t)$ in Red, and $x(t)$ in Blue. Bottom: Control $D(t)$ in Green.

are not necessarily monotone. In future work, we hope to use our approach to study other kinetic laws, or models with several substrates, biomasses, or reactions that contain uncertainties; see Beauthier et al. (2015) and Gouzé and Robledo (2006) for undelayed versions of such systems.

\section{Appendices}

\section{Appendix A: Proof of Lemma 1}

We build functions $\mu_{1}$ and $\gamma$ satisfying the requirements of Lemma 1 . Let $\varepsilon \in\left(0, s_{M} / 2\right)$ be any constant such that $\varepsilon \max \left\{\mu^{\prime}(\ell): 0 \leq \ell \leq s_{M}\right\}<2 \mu\left(s_{M} / 2\right)$. Choose

$$
\begin{aligned}
\mu_{1}(s)= & \left(\max \left\{0, s-s_{M}+\varepsilon\right\}\right)^{2} \\
& + \begin{cases}\mu(s), & 0 \leq s \leq s_{M} \\
2 \mu\left(s_{M}\right)-\mu(s), & s>s_{M}\end{cases}
\end{aligned}
$$

and $\gamma(s)=\left(\mu_{1}(s) / \mu(s)\right)-1$ for all $s>0$ and $\gamma(s)=0$ for all $s \leq 0$. If $s \leq s_{M}-\varepsilon$, then $\gamma(s)=0$. If $s \in\left(s_{M}-\varepsilon, s_{M}\right]$, 
then $\mu^{\prime}(s) \geq 0$, so

$$
\begin{aligned}
\gamma^{\prime}(s)= & \frac{1}{\mu^{2}(s)}\left[\mu(s)\left(2\left(s-s_{M}+\varepsilon\right)+\mu^{\prime}(s)\right)\right. \\
& \left.-\left(\left(s-s_{M}+\varepsilon\right)^{2}+\mu(s)\right) \mu^{\prime}(s)\right] \\
= & \frac{1}{\mu^{2}(s)}\left(s-s_{M}+\varepsilon\right)[2 \mu(s) \\
& \left.-\left(s-s_{M}+\varepsilon\right) \mu^{\prime}(s)\right] \\
\geq & \frac{1}{\mu^{2}(s)}\left(s-s_{M}+\varepsilon\right)\left[2 \mu\left(s_{M} / 2\right)\right. \\
& \left.-\varepsilon \max \left\{\mu^{\prime}(\ell): 0 \leq \ell \leq s_{M}\right\}\right]>0 .
\end{aligned}
$$

If $s>s_{M}$, then $\mu^{\prime}(s) \leq 0$, so since $s_{M}$ maximizes the function $\mu$, we have $\mu\left(s_{M}\right) \geq \mu(s)>0$ and so also

$$
\begin{aligned}
\gamma^{\prime}(s)= & \frac{1}{\mu^{2}(s)}\left[\mu(s)\left[2\left(s-s_{M}+\varepsilon\right)-\mu^{\prime}(s)\right]\right. \\
& \left.-\left[2 \mu\left(s_{M}\right)-\mu(s)+\left(s-s_{M}+\varepsilon\right)^{2}\right] \mu^{\prime}(s)\right]>0,
\end{aligned}
$$

so $\gamma$ is nondecreasing. Hence, we can choose $\mu_{1}$ and $\gamma$.

\section{Appendix B: Finishing the Proof of Theorem 1}

We convert the decay estimate (46) into the final ISS estimate. Since $s(t) \in(0, \bar{s})$ for all $t \geq 0$, it follows from the structure of our $z(t)$ dynamics in (14) and the fact that $\gamma$ is nondecreasing that the time derivative of

$$
V(z)=\frac{c_{g}(1+\gamma(\bar{s}))}{\mu_{1}\left(s_{*}\right)} z^{2}
$$

satisfies $\dot{V} \leq-2 c_{g} z^{2}(t)$ along all trajectories of the $z$ dynamics. Therefore, the time derivative of $\mathcal{U}_{3}\left(s_{t}, z(t)\right)=$ $\mathcal{U}_{2}\left(s_{t}\right)+V(z(t))$ satisfies

$$
\begin{aligned}
\dot{\mathcal{U}}_{3}(t) & \leq-c_{f} \mathcal{U}_{2}\left(s_{t}\right)-c_{g} z^{2}(t)+c_{g} \delta^{2}(t) \\
& \leq-c_{h} \mathcal{U}_{3}\left(s_{t}, z(t)\right)+c_{g} \delta^{2}(t)
\end{aligned}
$$

for all $t \geq \mathcal{T}_{d}(|X(0)|)$, where $c_{h}=\min \left\{c_{f}, \mu_{1}\left(s_{*}\right) /(1+\right.$ $\gamma(\bar{s}))\}$. Hence, applying an integrating factor and integrating over values $t \geq \mathcal{T}_{d}(|X(0)|)$ gives

$$
\begin{aligned}
& \mathcal{U}_{3}\left(s_{t}, z(t)\right) \leq \frac{c_{g}}{c_{h}}|\delta|_{[0, t]}^{2} \\
& +e^{-c_{h}\left(t-\mathcal{T}_{d}(|X(0)|)\right)} \mathcal{U}_{3}\left(s_{\mathcal{T}_{d}(|X(0)|)}, z\left(\mathcal{T}_{d}(|X(0)|)\right)\right)
\end{aligned}
$$

for all $t \geq \mathcal{T}_{d}(|X(0)|)$. Also, (15), (18), and (44) provide a function $\tau_{e} \in \mathcal{K}_{\infty}$ and a constant $\underline{u}>0$ such that

$$
\begin{aligned}
& \mathcal{U}_{3}\left(s_{\mathcal{T}_{d}(|X(0)|)}, z\left(\mathcal{T}_{d}(|X(0)|)\right)\right) \leq \tau_{e}(|X(0)|) \text { and } \\
& \mathcal{U}_{3}\left(s_{t}, z(t)\right) \geq \underline{u}\left(\left(s(t)-s_{*}\right)^{2}+z^{2}(t)\right) \geq \underline{u}|X(t)|^{2} / 3
\end{aligned}
$$

for all $t \geq \mathcal{T}_{d}(|X(0)|)$, where we used the fact that the second component $X_{2}$ of the error vector $X$ satisfies $\left|X_{2}(t)\right|^{2}=$ $\left|x(t)-\left(s_{\text {in }}-s_{*}\right)\right|^{2}=\left|s_{\text {in }}-s(t)-z(t)-\left(s_{\text {in }}-s_{*}\right)\right|^{2} \leq 2((s(t)-$ $\left.\left.s_{*}\right)^{2}+z^{2}(t)\right)$, and the bound $(\dot{s}(m))^{2} \leq 2\left(D(m) s_{\text {in }}\right)^{2}+$ $2\left(D(m)+(1+\bar{d}) \mu\left(s_{\text {in }}\right)\right)^{2}\left(|z(0)|+s_{\text {in }}\right)^{2}$ for all $m \geq 0$ to upper bound the integral part of $\mathcal{U}_{2}$. Hence, (A.5) and the fact that $\sqrt{a+b} \leq \sqrt{a}+\sqrt{b}$ for all $a \geq 0$ and $b \geq 0$ give

$$
\begin{aligned}
|X(t)| \leq & \sqrt{\frac{3}{\underline{u}} \tau_{e}(|X(0)|) e^{c_{h} \mathcal{T}_{d}(|X(0)|)}} e^{-t c_{h} / 2} \\
& +\sqrt{\frac{3 c_{g}}{\underline{u} c_{h}}}|\delta|_{[0, t]} \text { for all } t \geq \mathcal{T}_{d}(|X(0)|) .
\end{aligned}
$$

On the other hand, (15), (18), and the triangle inequality provide a continuous strictly increasing positive valued function $\mathcal{L}_{0}$ and a function $\mathcal{L}_{1} \in \mathcal{K}_{\infty}$ such that

$$
|\dot{X}(t)| \leq \mathcal{L}_{0}(|X(0)|)+\mathcal{L}_{1}\left(|\delta|_{[0, t]}\right)
$$

for all $t \in\left[0, \mathcal{T}_{d}(|X(0)|)\right]$. Integrating (A.7) and using the triangle inequality give

$$
\begin{aligned}
|X(t)| \leq & |X(0)| \\
& +\mathcal{T}_{d}(|X(0)|)\left(\mathcal{L}_{0}(|X(0)|)+\mathcal{L}_{1}\left(|\delta|_{[0, t]}\right)\right) \\
\leq & e^{\mathcal{T}_{d}(|X(0)|)-t} \mathcal{R}_{1}(|X(0)|)+\mathcal{R}_{2}\left(|\delta|_{[0, t]}\right)
\end{aligned}
$$

for all $t \in\left[0, \mathcal{T}_{d}(|X(0)|)\right]$, where $\mathcal{R}_{1}(p)=p+\mathcal{T}_{d}(p) \mathcal{L}_{0}(p)+$ $\frac{1}{2} \mathcal{T}_{d}^{2}(p)$ and $\mathcal{R}_{2}(p)=\frac{1}{2} \mathcal{L}_{1}^{2}(p)$ are both of class $\mathcal{K}_{\infty}$. The final ISS estimate now follows by adding (A.6) and (A.8).

\section{References}

Beauthier, C., J. Winkin and D. Dochain (2015). Input/state invariant LQ-optimal control: application to competitive coexistence in a chemostat. Evolution Equations and Control Theory 4(2), 143-158.

Bernard, O., Z. Hadj-Sadok, D. Dochain, A. Genovesi and J.-P. Steyer (2001). Dynamical model development and parameter identification for an anaerobic wastewater treatment process. Biotechnology and Bioengineering 75(4), 424-438.

Cougnon, P., D. Dochain, M. Guay and M. Perrier (2011). On-line optimization of fedbatch bioreactors by adaptive extremum seeking control. Journal of Process Control 21(10), 1526-1532.

Fridman, E., A. Seuret and J.-P. Richard (2004). Robust sampled-data stabilization of linear systems: an input delay approach. Automatica 40(8), 1441-1446.

Fritsch, C., J. Harmand and F. Campillo (2015). A modeling approach of the chemostat. Ecological Modelling 299, 1-13.

Gouzé, J.-L. and G. Robledo (2005). Feedback control for nonmonotone competition models in the chemostat. Nonlinear Analysis: Real World Applications 6(4), 671690.

Gouzé, J.-L. and G. Robledo (2006). Robust control for an uncertain chemostat model. International Journal of Robust and Nonlinear Control 16(3), 133-155.

Khalil, H. (2002). Nonlinear Systems, Third Edition. Prentice Hall. Upper Saddle River, NJ.

Lemesle, V. and J.-L. Gouzé (2008). A simple unforced oscillatory growth model in the chemostat. Bulletin of Mathematical Biology 70(2), 344-357.

Mathematica (2015). The world's definitive system for modern technical computing. Wolfram. Accessed August 18, 2016. http://www.wolfram.com/mathematica/.

Mazenc, F. and M. Malisoff (2010). Stabilization of a chemostat model with Haldane growth functions and a delay in the measurement. Automatica 46(9), 14281436.

Mazenc, F., J. Harmand and H. Mounier (2013a). Global stabilization of the chemostat with delayed and sampled measurements and control. In Proceedings of the 9th IFAC Symposium on Nonlinear Control Systems. 
Toulouse, France. pp. 170-174.

Mazenc, F., J. Harmand and M. Malisoff (2016). Stabilization in a chemostat with sampled and delayed measurements. In Proceedings of the American Control Conference. Boston, MA. pp. 1857-1862.

Mazenc, F., M. Malisoff and J. Harmand (2008). Further results on stabilization of periodic trajectories for a chemostat with two species. IEEE Transactions on Automatic Control 53(Special Issue on Systems Biology), 6674.

Mazenc, F., M. Malisoff and T. Dinh (2013b). Robustness of nonlinear systems with respect to delay and sampling of the controls. Automatica 49(6), 1925-1931.

Monod, J. (1950). La technique de culture continue: Théorie et applications. Annales de l'Institut Pasteur 79, 390-410.

Novick, A. and L. Szilard (1950). Description of the chemostat. Science 112(2920), 715-716.

Robledo, G. (2009). Feedback stabilization for a chemostat with delayed output. Mathematical Biosciences and Engineering 6(3), 629-647.

Robledo, G., F. Grognard and J.-L. Gouzé (2012). Global stability for a model of competition in the chemostat with microbial inputs. Nonlinear Analysis: Real World Applications 13(2), 582-590.

Smith, H. and P. Waltman (1995). The Theory of the Chemostat. Cambridge University Press. Cambridge, UK. 